\title{
Genetic effects on planum temporale asymmetry and their limited relevance to neurodevelopmental disorders, intelligence or educational attainment
}

\author{
Amaia Carrion-Castillo ${ }^{a}$, Antonietta Pepe ${ }^{b}$, Xiang-Zhen Kong ${ }^{a}$, \\ Simon E. Fisher ${ }^{a, c}$, Bernard Mazoyer ${ }^{b}$, Nathalie Tzourio-Mazoyer ${ }^{b}$, \\ Fabrice Crivello ${ }^{b}$ and Clyde Francks ${ }^{a, c}, * *$ \\ ${ }^{a}$ Language and Genetics Department, Max Planck Institute for Psycholinguistics, Nijmegen, the Netherlands \\ ${ }^{\mathrm{b}}$ Groupe d'Imagerie Neurofonctionnelle, Institut des Maladies Neurodégénératives, Centre National de la Recherche \\ Scientifique, Commissariat à l'Energie Atomique, et Université; de Bordeaux, Bordeaux, France \\ ${ }^{c}$ Donders Institute for Brain, Cognition and Behaviour, Radboud University, Nijmegen, the Netherlands
}

\section{A R T I C L E I N F O}

Article history:

Received 8 May 2019

Reviewed 1 September 2019

Revised 1 November 2019

Accepted 13 November 2019

Published online 29 November 2019

Keywords:

Planum temporale

UK biobank

Genome-wide association

Genetic correlation

Neurodevelopmental disorders

Brain asymmetry

\begin{abstract}
A B S T R A C T
Previous studies have suggested that altered asymmetry of the planum temporale (PT) is associated with neurodevelopmental disorders, including dyslexia, schizophrenia, and autism. Shared genetic factors have been suggested to link PT asymmetry to these disorders. In a dataset of unrelated subjects from the general population (UK Biobank, $\mathrm{N}=18,057$ ), we found that PT volume asymmetry had a significant heritability of roughly $14 \%$. In genome-wide association analysis, two loci were significantly associated with PT asymmetry, including a coding polymorphism within the gene ITIH5 that is predicted to affect the protein's function and to be deleterious (rs41298373, $p=2.01 \times 10-15$ ), and a locus that affects the expression of the genes BOK and DTYMK (rs7420166, $p=7.54 \times 10-10$ ). DTYMK showed left-right asymmetry of mRNA expression in post mortem PT tissue. Cortex-wide mapping of these SNP effects revealed influences on asymmetry that went somewhat beyond the PT. Using publicly available genome-wide association statistics from large-scale studies, we saw no significant genetic correlations of PT asymmetry with autism spectrum disorder, attention deficit hyperactivity disorder, schizophrenia, educational attainment or intelligence. Of the top two individual loci associated with PT asymmetry, rs41298373 showed a tentative association with intelligence (unadjusted $p=.025$ ), while the locus at BOK/DTYMK showed tentative association with educational attainment (unadjusted Ps $<.05$ ). These findings provide novel insights into the genetic contributions to human brain asymmetry, but do not support a substantial polygenic association of PT
\end{abstract}

\footnotetext{
* Corresponding author. Max Planck Institute for Psycholinguistics, Po Box 310, 6500 AH, Nijmegen, the Netherlands.

E-mail address: clyde.francks@mpi.nl (C. Francks).
} 
asymmetry with cognitive variation and mental disorders, as far as can be discerned with current sample sizes.

(C) 2019 Elsevier Ltd. All rights reserved.

\section{Introduction}

The planum temporale (PT) is a triangular-shaped region of the cerebral cortex, located posteriorly on the superior temporal gyrus (STG) (Altarelli, et al., 2014). Geschwind and Levitsky (1968) first described left-right asymmetry of PT surface area fifty years ago. They analysed 100 post-mortem brains and observed a leftward asymmetry (left > right) in 65 of them, rightward asymmetry in 10, and approximate symmetry in 24 . Re-analysis of the same brains emphasised that this variation is continuous in nature (Galaburda, Corsiglia, Rosen, \& Sherman, 1987). Further studies have consistently shown that the PT is leftward lateralized in the general population, and that the left PT can be up to 50\% larger than the right, although the precise anatomical definition affects this estimate (Toga \& Thompson, 2003; Tzourio-Mazoyer et al., 2010, 2019; Tzourio-Mazoyer \& Mazoyer, 2017; Watkins et al., 2001).

Lesion and functional studies suggest that the PT is implicated in a number of language-related processes, including auditory and phonological processing, and language comprehension (Dronkers, Wilkins, Van Valin, Redfern, \& Jaeger, 2004). Initial reports proposed that PT asymmetry may be an anatomical marker for left-hemisphere dominance for language processing (Foundas, Leonard, Gilmore, Fennell, \& Heilman, 1994; Geschwind \& Levitsky, 1968). However, recent studies have found that there is no correlation between PT asymmetry and functional language lateralization as assessed at the whole hemisphere level (Keller et al., 2011; Tzourio-Mazoyer, Crivello, \& Mazoyer, 2018). Rather, it seems that PT anatomical asymmetry is associated with functional lateralization only locally, in directly neighbouring auditory areas (Tzourio-Mazoyer, Crivello, \& Mazoyer, 2018).

Alterations of the typical form of PT asymmetry have been reported in dyslexia. In a post-mortem analysis, Galaburda, Sherman, Rosen, Aboitiz, and Geschwind (1985) observed symmetrical plana temporale in the brains of all dyslexic individuals that they studied (4 males and 3 females), but subsequent studies using neuroimaging have found contradictory results (see Table 1 in Altarelli et al. (2014) for an overview). Using magnetic resonance imaging (MRI), Altarelli et al. (2014) manually outlined the PT according to the same anatomical criteria as in earlier post mortem studies, in 35 dyslexic cases and 46 controls. They found an increased proportion of rightward surface area asymmetry among dyslexic boys specifically, and suggested that previous mixed results may have arisen due to different anatomical definitions of the PT, and not accounting adequately for sex.

A reduction of PT grey matter volume asymmetry has also been reported in people with autism spectrum disorder (ASD), with a smaller left PT in cases causing a change of asymmetry (Rojas, Bawn, Benkers, Reite, \& Rogers, 2002; Rojas, Camou, Reite, \& Rogers, 2005). A reduction of the left PT has been proposed to relate to delays in language acquisition, which can occur in some ASD patients (Knaus, Kamps, Foundas, \& Tager-Flusberg, 2018). Consistent with this, a recent study of brain regional growth rates in newborns with congenital heart diseases reported a strong correlation between the growth rate of left PT and language scores at 12 months of age (Jakab et al., 2019). Furthermore, a meta-analysis of ten studies concluded that a reduction of PT area asymmetry is a feature of schizophrenia (Sommer, Ramsey, Kahn, Aleman, \& Bouma, 2001), although it is not clear whether this change in asymmetry is driven by a reduced left PT or increased right PT in cases, or both ((Hasan et al., 2011; Hirayasu et al., 2000; Shapleske, Rossell, Woodruff, \& David, 1999)). A reduction of PT asymmetry in terms of area and thickness measures has also been observed in schizophrenia (SCZ) (Ratnanather et al., 2013). Auditory hallucinations can occur in SCZ (MorchJohnsen et al., 2017), which may conceivably relate to PT functions as secondary auditory cortex (Crow, Ball, Bloom, \& al, 1989).

Average leftward PT asymmetry is established early in development, as evidenced by studies of fetuses (Chi, Dooling, \& Gilles, 1977), preterm newborns (Dubois, et al., 2010) and infants (Li et al., 2014; Wada, Clarke, \& Hamm, 1975). A developmental program that results in population-level average asymmetry is likely to require a molecular genetic component that first creates a directional left-right axis early in development (Francks, 2015; Postema, Carrion-Castillo, Fisher, Vingerhoets, \& Francks, 2019; Reiter \& Leroux, 2017; Willems, Van der Haegen, Fisher, \& Francks, 2014). This is true for the visceral organs, for which asymmetry arises from a well-regulated genetic-developmental pathway, originating with molecular chirality in the very early embryo (Deng, Xia, \& Deng, 2015; Norris, 2012). Thus, we regard population-level leftward average asymmetry of the PT as being necessarily encoded in the human genome, but by mechanisms that are currently unknown.

It is also largely unknown which factors affect interindividual variability of PT asymmetry around the population's leftward average, or to what extent these factors might be heritable. Shared genetic contributions to schizophrenia and PT asymmetry have long been postulated (Crow, 1993; Sommer, Ramsey, Kahn, Aleman, \& Bouma, 2001), although early hypotheses were formulated around single genetic loci of large effect, and it is now undisputed that there is a substantial polygenic contribution to schizophrenia, with significant SNP-based heritability and multiple susceptibility loci identified (Ripke et al., 2014). A shared genetic contribution to PT asymmetry and dyslexia has also been suggested, on the basis that pre-readers and adolescents at familial risk of dyslexia have shown a reduced leftward PT asymmetry compared to those with no familial risk (Vanderauwera et al., 2018). 
Table 1 - Significantly associated loci with PT asymmetry in the UK Biobank dataset.

\begin{tabular}{llllllllllllllllll}
\hline Rsid & Chr & Pos & A0 & A1 & AF & INFO & HWE & T & P & $\begin{array}{c}\text { Context } \\
\text { Nearest } \\
\text { Gene }\end{array}$ & Dist & CADD & RDB & Min Common \\
\hline rs7420166 & 2 & 242506733 & G & A & .26 & .95 & .70 & 6.16 & $7.54 \times 10^{-10}$ & intronic & BOK & 0 & 1.86 & 7 & 11 & 11 & \\
rs41298373 & 10 & 7622009 & G & A & .10 & 1.00 & .81 & 7.958 & $2.01 \times 10^{-15}$ & nonsyn & ITIH5 & 0 & 27.90 & 5 & 4 & 15 \\
\hline
\end{tabular}

Rsid: Rs identity of the lead SNP within the locus. Pos: chromosome position of the SNP in the hg19 human reference genome. A0 and A1 are the SNP alleles. A1 is the 'effect allele', i.e., the direction of the association T statistic with the PT AI indicates the additive effect of each A1 allele. AF: allele frequency of the effect allele. INFO: imputation quality score. HWE: Hardy-Weinberg $p$-value. T: association T-statistic. P: association $p$ value. Context: location of the SNP with respect to the nearest gene, and consequence at the protein level if exonic (nonsyn: non synonymous). Dist: distance to the nearest gene in basepairs. CADD: Combined Annotation Dependent Depletion (CADD) score (scores greater than 15 indicate deleterious SNPs). RDB: regulome DB categorical score (from 1a to 7). 1a is the highest score for SNPs with the most biological evidence to be a regulatory element. Chrom min and chrom common: the minimum and most common chromatin states across 127 tissue/cell types. States are described using a 15 point scale, with the lower the chromatin score the greater the accessibility to the genome at this site (scores of less than 8 indicate an open chromatin region)

A previous genome-wide association scan (GWAS) metaanalysis for PT volume asymmetry, in just over 3,000 healthy adult subjects, did not find individual loci which were significant after correcting for multiple testing over the whole genome (Guadalupe et al., 2015). However, a significant enrichment of association signals was found within sets of genes with roles in steroid hormone receptor activity and steroid metabolic processes. Gene sets involved in steroid hormone biology were tested because PT asymmetry showed the most significant sex difference of all cortical regional asymmetries, with males more leftward asymmetric on average (Guadalupe et al., 2015). It has also been reported that foetal testosterone levels relate to the development of grey matter asymmetries of some cortical regions, including the PT (Lombardo et al., 2012). In fact, an extensive and influential theory was developed by Geschwind and Galaburda which involves sex hormones, brain laterality and disorder susceptibility (Geschwind \& Galaburda, 1985), which still motivates current research, although definitive support is lacking (Bryden, McManus, \& Bulman-Fleming, 1994; Hollier, Maybery, Keelan, Hickey, \& Whitehouse, 2014; Papadatou-Pastou \& Martin, 2017).

In the present study, we used the UK Biobank MRI dataset $(\mathrm{N}=18,057)$ to perform a more highly powered genetic analysis of PT asymmetry than has previously been possible. This sample size also permitted, for the first time, the estimation of SNP-based heritability of PT asymmetry, and its genetic correlations with other traits. Our goals were (i) to identify individual genetic loci and gene sets related to variation in PT asymmetry, which can provide novel insights into genetic contributions to brain laterality; (ii) for any significant loci arising from GWAS of PT asymmetry, to map their potential effects on cerebral cortical asymmetry more broadly; (iii) to examine whether polygenic influences on PT asymmetry also impact cognitive traits and psychiatric disorders. For the latter purpose we made use of summary statistics from large-scale GWAS studies of ASD and schizophrenia. There are currently no large-scale GWAS results available for dyslexia (based on sample sizes of over 10,000), but we also used GWAS summary statistics for intelligence and educational attainment (EA), as these traits are correlated both phenotypically and genetically with language and reading abilities in the general population (Verhoef et al., 2019). Finally we included analysis of GWAS summary statistics for attention deficit/ hyperactivity disorder (ADHD), as this disorder is often comorbid with dyslexia and negatively genetically correlated with reading measures (Verhoef et al., 2019).

\section{Materials and methods}

None of the study procedures or analyses were pre-registered prior to this research being conducted. We report how we determined our sample size, all data exclusions, all inclusion/ exclusion criteria, whether inclusion/exclusion criteria were established prior to data analysis, all manipulations, and all measures in the study.

\subsection{Dataset}

Data were obtained from the UK Biobank cohort (https://www. ukbiobank.ac.uk/using-the-resource/), as part of research application 16066, with Clyde Francks as the principal applicant. This is a general adult population cohort. The data collection in the UK Biobank, including the consent procedure, has been described elsewhere (Sudlow et al., 2015). Informed consent was obtained by the UK Biobank for all participants. For this study we used data from the October 2018 release of 22,392 participants' brain MRI data, together with the genomewide genetic data from the same participants. The age range of these participants was from 44 to 80 years (median 63.9), and 11,269 were female, 10,551 were male.

\subsection{PT volume and asymmetry measures}

The UK Biobank provided various derived brain imaging measures (Miller et al., 2016) and documentation (https:// biobank.ctsu.ox.ac.uk/crystal/crystal/docs/brain_mri.pdf).

Briefly, tissue-type segmentation (Zhang, Brady, \& Smith, 2001) was performed from $\mathrm{T} 1$ images using FSL FAST (Jenkinson, Beckmann, Behrens, Woolrich, \& Smith, 2012; Smith et al., 2004), and 44 cerebral cortical regions were defined per hemisphere based on the Harvard-Oxford probability atlas (distributed with the FSL software package; http:// fsl.fmrib.ox.ac.uk/fsl/). For the present study we focus on the PT as defined in the Harvard-Oxford atlas (Fig. 1), which makes use of an anatomical definition close to that used in the original post mortem studies of this region (see Introduction). 


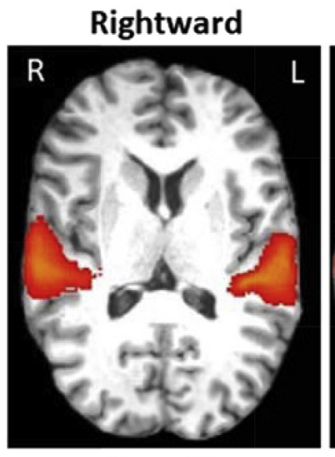

$A I=-.48$
Equal volume

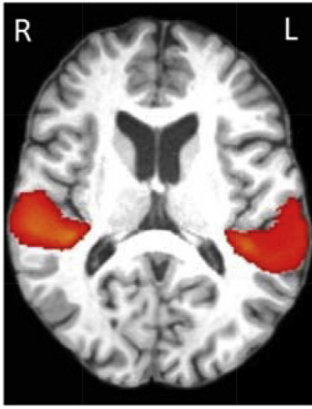

$A I=0$

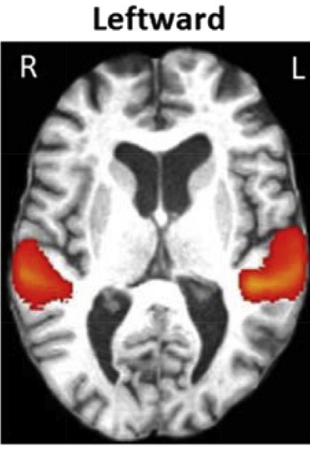

$A I=.50$

\section{Extreme Leftward}



$A l=.99$

Fig. 1 - Examples of four subjects with different PT grey matter volume asymmetries. Transverse brain images are shown. The left and right PT are indicated according the Harvard-Oxford atlas definitions.

Grey matter volumes of the left and right PT were calculated per subject by summing the voxel-wise grey matter volume estimates within each region.

We extracted the left (L) and right (R) PT grey matter volumes (UKB field IDs = 25872 and 25873), and calculated an asymmetry index (AI) per individual subject as (L-R)/((L+R)/2). Given this definition, a positive AI reflects a leftward asymmetry of PT grey matter volume. The main focus of the present study was on the AI, but the L and R measures separately, as well as total brain volume (TBV, UKB field ID: 25010, 'Volume of brain, grey + white matter') were also analysed in some contexts, to aid the interpretation of findings involving the AI. All analyses, data transformations and filtering steps were done in $\mathrm{R}$ (version 3.3.2).

Outliers were removed at greater than 4 SD from the mean (calculated after the exclusion of some subjects on the basis of genetic quality control, see below) separately for each trait (L, $\mathrm{R}, \mathrm{AI}$, and TBV). For each trait we then performed linear regression, using the $I m$ function in $\mathrm{R}$, and extracted the residuals after correcting for sex, age, nonlinear age (zage2), genotyping array, scanner position parameters ( $\mathrm{x}=$ lateral, $\mathrm{y}=$ transversal, $\mathrm{z}=$ longitudinal), and the first ten principal components (PCs) which capture genome-wide population structure in the genotype data and were made available by the UK Biobank (Bycroft et al., 2018) (Table S1). The distribution of each trait was visually inspected before and after residualizing (Fig. S1). The outlier exclusion, together with some missing PT data in the UK Biobank release, and some missing or outlier subjects for some covariates, meant that between 18,037 and 18,053 subjects were available with both post-QC genetic data and PT or TBV measures, depending on the specific measure (Table S2).

\subsection{Heritability and genetic correlations between PT measures and total brain volume}

Subject-level genetic quality control is described in the Supplementary Methods. Genome-based restricted maximum likelihood (GREML) (Yang et al., 2010) analyses were performed to estimate the SNP-based heritabilities $\left(\mathrm{h}^{2}{ }_{\mathrm{SNP}}\right)$ of the AI, $\mathrm{L}, \mathrm{R}$, and TBV measures using GCTA (version 1.26.0). Bivariate GREML was used to estimate genetic correlations between measures. See the Supplementary Methods for details.

\subsection{Genome wide association scanning}

After SNP-level quality control (see Supplementary Methods) there were 15,120,452 SNPs available for GWAS. GWAS was performed separately for each of the residualized PT AI, L and $R$ measures, using imputed genotype dosages and an additive model, with BGENIE (v1.2) (Bycroft et al., 2018). Sample sizes ranged from 18,037 to 18,053 depending on the specific measure (Table S2). Manhattan plots of the results, and QQplots, were made using the "qqman" $\mathrm{R}$ (Turner, 2017) package, and regional association plots were made using LocusZoom v1.4 (Pruim et al., 2010).

The summary statistics from the GWAS for PT AI were loaded into the annotation software FUMA (Watanabe, Taskesen, van Bochoven, \& Posthuma, 2017). Significantly associated SNPs were considered as those with pointwise $p<5 \times 10-8$, to correct for multiple testing over the whole genome. These SNPs were annotated functionally with respect to various bioinformatic databases (see Supplementary Methods).

\subsection{Gene-set enrichment analysis}

Gene-set enrichment analysis was applied to the GWAS results for the PT AI, using the software MAGMA v1.06b (de Leeuw, Mooij, Heskes, \& Posthuma, 2015). The basis of this analysis is to first assign a single association score to each gene based on the GWAS data, and then test whether the genes in a given set show, on average, more evidence for association with the trait in question than the rest of the genes in the genome, for which scores could be calculated (hence this is sometimes called 'competitive' analysis). We tested for enrichment according to the Gene Ontology (GO) classification system (Ashburner et al., 2000; GO Consortium, 2017). A total of 5,875 GO sets (with a minimum of 10 genes per set) were tested, and Bonferroni multiple testing correction was applied to obtain corrected $p$-values. See Supplementary Methods for details.

We also looked up the MAGMA enrichment results, without correcting for multiple testing, for two specific GO gene-sets related to steroid hormone biology ('steroid hormone receptor activity' and 'steroid metabolic process') which were enriched for association signals with PT asymmetry in a previous, smaller GWAS study of PT asymmetry (see 
Introduction) (Guadalupe et al., 2015). For these two gene sets only, we also ran a different type of enrichment analysis called INRICH (Lee, OD;ushlaine, Thomas, \& Purcell, 2012), that had been used in the previous study by Guadalupe et al. (2015), in order to understand whether differences in the results between the present study and the previous study might be due to this. INRICH settings were the same as used by Guadalupe et al. (2015); one of the settings is an association $p$ value cut-off for including SNPs from the GWAS, which was set at .001 (whereas MAGMA avoids this kind of threshold setting and was therefore preferred for the main enrichment analysis in the present study).

\subsection{Further analysis of rs41298373 and rs7420166}

Two SNPs, rs41298373 and rs7420166, were subject to further characterisation and analysis because they were significantly associated with the PT AI in the GWAS. Rs41298373 is a protein coding variant with a predicted deleterious impact, located in the gene ITIH5, while rs7420166 was the lead SNP at the chromosome 2q37 locus, located within an intron of the gene BOK (see Results).

\subsubsection{Bioinformatic characterisation}

For rs41298373 and rs7420166, we assessed evolutionary conservation and expression QTL (eQTL) effects (see Supplementary Methods). These analyses would implicate BOK but also another nearby gene, DTYMK (see Results). We further queried the spatio-temporal expression patterns of the genes ITIH5, BOK, and DTYMK using publicly available data (Supplementary Methods).

Using the Allen Brain Atlas microarray data, which are based on six post mortem brains (only two have data from both hemispheres), we also assessed whether the expression of ITIH5, BOK or DTYMK in the PT differed between hemispheres, by modelling expression as a function of probe and hemisphere, and including subject as a random factor [expression $\sim$ (1|subject) + probe + hemisphere], using the lmer function in the 'lme4' package in R. We used all data assigned to the region 'PLT' as defined in the Allen data.

\subsubsection{Brain-wide effects on grey matter asymmetry}

The SNPs rs41298373 and rs7420166 were detected in GWAS for PT asymmetry using an atlas-based definition of this cortical region (see above), for which the atlas definition itself is asymmetrical (see Discussion for more on this issue). We therefore performed grey matter voxel-based-morphometry (VBM) association analyses for each of the SNPs rs41298373 and rs7420166, to confirm that the two loci affect PT asymmetry in a complementary methodological context, and to assess their potential effects on asymmetry brain-wide. For this purpose we required a symmetrized template and no regional atlas definitions. Briefly, voxel-wise grey matter volume was assessed with FSLVBM (Douaud et al., 2007) (http://fsl.fmrib.ox.ac.uk/fsl/fslwiki/FSLVBM) carried out with FSL (v5.0) tools (Jenkinson, Beckmann, Behrens, Woolrich, \& Smith, 2012; Smith et al., 2004). Subject's grey matter images were non-linearly normalized onto a leftright-symmetric grey matter template in the standard MNI 152 space. The generated maps were smoothed with isotropic
Gaussian kernel (sigma $=2.55$, full-width at halfmaximum $=6 \mathrm{~mm}$ ). Next, voxel-wise asymmetry index (AI) maps were calculated using the formula $(L-R) /((L+R) / 2)$ for each pair of left-right corresponding voxels, per subject. Finally, we ran association analyses of rs41298373 and rs7420166 with each voxel AI using mri_glmfit (http://surfer. nmr.mgh.harvard.edu/fswiki/mri_glmfit) from FreeSurfer (v6.0) (Dale, Fischl, \& Sereno, 1999; Fischl, Sereno, \& Dale, 1999), including the covariates listed in Table S1, and testing the additive effect of each genotype (hard-called genotypes, call probability threshold .9, resulting in $\mathrm{N}=18052$ subjects for rs41298373 and $\mathrm{N}=16663$ for rs7420166). Multiple comparison correction was performed on statistical maps using the 3dClustSim program implemented in AFNI (version 16.2.19). Thresholds of voxel-level $p<.001(\mathrm{Z}=3.29)$ and cluster-level $p<.01$ (cluster size $\geq 40$ voxels) were used based on Monte Carlo simulation in the left hemisphere mask. Results were mapped to left-hemisphere surface space for visualization of cerebral cortical effects, using BrainNet Viewer (v1.63) (Xia, Wang, \& He, 2013) (the 'extremum voxel' option).

\subsubsection{Vertex-wise analysis of rs41298373 and rs7420166} We used cortical surface-based analysis to investigate cortexwide, vertex-wise effects of rs41298373 and rs7420166, again without regard to any regional atlas definitions, and using a hemispheric co-registration approach to further improve interhemispheric correspondence. This analysis potentially offered greater sensitivity to detect subtle asymmetrical effects of the two loci.

The analysis used a subset of 8,778 subjects (from an earlier release of UK Biobank data) for which cortical surface extraction was obtained using FreeSurfer (v6.0) (Dale et al., 1999; Fischl et al., 1999) (https://surfer.nmr.mgh.harvard. edu/) with $1 \mathrm{~mm}^{3} \mathrm{~T} 1$ and FLAIR acquisitions as inputs. A quality control procedure (ENIGMA Consortium protocol, http://enigma.ini.usc.edu) for FreeSurfer surface reconstruction was performed on the cortical surface area and cortical thickness estimates at both hemispheric and regional (Desikan et al., 2006) levels. This involved producing interactive web-page reports for group-level statistics of the hemispheric and regional measures, and subject-specific web-page reports for surface reconstruction accuracy. Visual inspection of the subject-specific reports was performed on individuals flagged as possible outliers (if they deviated more than 4 SD from the mean) based on the group-level statistics of any of the aforementioned measures, and resulted in the exclusion of 189 subjects.

The left and right cortical surfaces of the 8589 remaining participants were then warped into a left/right symmetrical standard space (fsaverage_sym) using a surface-based interhemispheric co-registration procedure acting at the vertex level (Maingault, Tzourio-Mazoyer, Mazoyer, \& Crivello, 2016; Greve et al., 2013). This inter-hemispheric alignment step was done twice: once on the left hemisphere of the symmetrical fsaverage_sym template, and once on its right hemisphere counterpart. In these two surface models, and for each participant, vertex-level cortical thickness and cortical surface area left-right difference (L-R) maps were generated. The two cortical thickness asymmetry maps (one 
for each hemisphere of fsaverage_sym surface model) were further averaged to produce a more robust left-right difference map, which was unbiased for positional structural asymmetries between hemispheres. The same was done for cortical surface area asymmetry. Again, group-based quality control of the inter-hemispheric vertex-wise alignment was performed, which led to a useable sample of 8578 subjects (11 additional subjects excluded for failure of inter-hemispheric registration).

Next, the cortical thickness and surface area vertex-wise left-right difference maps were smoothed with a $15 \mathrm{~mm}^{2}$ FWHM surface-based Gaussian kernel, and processed with the previously mentioned mri_glmfit Freesurfer commandline to search for possible associations of rs41298373 and rs7420166 with each vertex, using the same statistical model as in the VBM analysis (above), and covariates listed in Table S1. Coupled with the previous genetic quality control (see above) and the available demographic data, and testing the additive effect of each genotype (hard-called genotypes, call probability threshold .9), there were $\mathrm{N}=7231$ subjects for rs41298373 and $N=6685$ for rs7420166. Association maps with the two SNPs were obtained after cluster wise correction at $p<.05$ for multiple comparisons, by parametric Gaussian based Monte Carlo simulations, and .005 as the cluster forming threshold.

\subsection{Disorders, cognitive and behavioural traits}

\subsubsection{Phenotypic associations}

We tested whether self-reported handedness (UK Biobank field ID 1707, coded as right-handed $(\mathrm{N}=16,076)$ us lefthanded ( $N=1,703)$ and excluding ambilaterals) is associated with PT measures (L, R, AI). For this we used regression models in which a given PT measure was the dependent variable, and predictor variables were handedness, sex, scanner position variables, and linear and non-linear age (see Table S4). We also repeated this analysis including TBV as an additional predictor variable in each model.

The UK Biobank has a semi-quantitative measure of Fluid Intelligence (FI; field ID 1707; the sum of 13 items). In addition, we derived a measure of EA according to the approach by Lee et al. (2018), measured as the number of years of schooling that individuals completed (EduYears). The distributions of FI and EduYears are shown in Fig. S2. We separately tested each of FI and EduYears for association with the PT measures (L, R and $\mathrm{AI})$, again by multiple regression models using the same additional covariate effects as when for testing handedness (Table S4).

Within the subset of UK Biobank participants used for the present study (i.e., the subset with available brain MRI data), only 7 participants had an ICD-10 diagnosis of schizophrenia, and only one individual had a diagnosis of expressive language disorder. These numbers did not support analysis in relation to $\mathrm{PT}$ measures.

\subsubsection{Genome-wide genetic correlation}

We estimated genetic correlations of the PT AI, L and R measures with SCZ, ASD, ADHD, intelligence, and EA. This analysis was based on GWAS summary statistics for the PT measures as generated in this study, together with publicly available GWAS summary statistics for ASD (Grove et al., 2019), SCZ (Ripke et al., 2014), ADHD (Demontis et al., 2019), intelligence (Savage et al., 2018) and educational attainment (EA) (Lee et al., 2018). Sample sizes used for each of these GWAS, prevalence and other parameters are summarized in Table S5; we used the publicly available summary statistics of the largest-to-date GWASes for each of these traits.

We assessed genetic correlation using two different methods which are both designed to make use of GWAS summary statistics, i.e., do not require individual level data: LD score regression (LDSC, v.1.0.0) (Bulik-Sullivan et al., 2015) and SumHer (Speed \& Balding, 2019), the latter using both the LDAK and GCTA models (see Supplementary Methods).

We estimated the minimum power to detect genetic correlations across these pairs of traits and datasets at alpha $<.05$ using the GCTA-GREML power calculator (Visscher et al., 2014), given the sample sizes involved, and according to SNP-based heritability estimates obtained from LDSC and SumHer. Tables S3 and S5 summarize all the parameters per trait that went into the power calculations. Since LD score regression (Bulik-Sullivan et al., 2015) and SumHer (Speed \& Balding, 2019) utilize summary statistics while GCTA relies on the individual genotype data, the true power is likely to be slightly lower for the former two; however, the GCTA-GREML power calculator gives an indicative estimate.

2.7.3. Top SNPs and gene sets associated with PT asymmetry For three individually significant SNPs from the GWAS of PT AI in the UK Biobank (see Results), we looked up these SNPs in the publicly available GWAS results for ASD, SCZ, ADHD, intelligence and EA. In case the SNP was not present in the publicly available GWAS results, we identified proxy SNPs in high LD $\left(r^{2}>.8\right)$ with them, as calculated within the UKB imaging dataset using plink (v1.90).

\section{Results}

\subsection{PT asymmetry and its associations}

As expected, PT grey matter volume in the UK Biobank dataset of 18,049 healthy adult subjects, as measured using the Harvard-Oxford atlas, was leftward asymmetrical at the population level (Figs. S1, S3), with males having a slightly more pronounced leftward asymmetry than females $\left(\mathrm{t}=-11.38, p\right.$-value $=6.17 \times 10^{-30}$, Fig. S4 $)$.

The PT AI was not associated with handedness (Fig. S5, Table S4) or Fluid Intelligence (Table S4), but was nominally associated with EduYears (unadjusted $p=.037$, partial correlation -.016) (Table S4). This was due to EduYears being more strongly associated with the right PT volume than the left (Table S4). As the Biobank's Fluid Intelligence measure is fairly crude (sum of 13 items), the lack of phenotypic association with this measure did not discount a possible genetic correlation of PT asymmetry with intelligence, when analysing GWAS data from other studies of intelligence. We therefore continued to test for this genetic correlation using published GWAS summary statistics for intelligence (see further below).

Regarding the L and R measures separately, FI was associated with both $\mathrm{L}$ and $\mathrm{R}$, and both before and after adjustment 
for TBV (Table S4), so that this is a bilateral association. For handedness, there was an association with PT R that became a little stronger after adjusting for TBV (the lowest $p$ value in relation to handedness was .002 (unadjusted) for the TBVadjusted $\mathrm{R}$ measure, partial correlation .023; Table S4). However, there was also a trend of association between handedness and PT L that became significant after adjusting for TBV (Table S4), so that again this was a weak, bilateral association (while the association of handedness with PT AI was clearly not significant; Table S4; Fig. S5).

There was a negative relation bilaterally between PT L and $R$ volumes and increasing age, which was partly attenuated after adjusting for TBV (Fig. S6).

\subsection{Genetic architecture of PT asymmetry}

The PT AI showed a significant SNP-based heritability, as assessed with GCTA (Yang et al., 2010) $\left[\mathrm{h}^{2}{ }_{\mathrm{SNP}}(\mathrm{AI})=.14\right.$ se $\left.\left.=.035, p=1.38 \times 10^{-5}\right)\right]$. The SNP-based heritabilites for the left and right PT volumes, considered separately, were also significant and similar in magnitude to each other $\left[\mathrm{h}^{2}{ }_{\mathrm{SNP}}(-\right.$ left $)=.454, \mathrm{~h}^{2}$ SNP $($ right $\left.)=.414\right]$. However, the genetic correlation between left and right PT volumes was significantly different from $1\left[\rho_{(\mathrm{L}, \mathrm{R})}=.85\right.$ (95\% CI .74-.98), $p=.009$, null hypothesis $=$ different to 1$]$, that indicates that there are some genetic effects which are not shared between the left and right PT, and therefore constitute heritable contributions to PT asymmetry. There was a low but significant phenotypic correlation between $\mathrm{AI}$ and $\mathrm{TBV}\left(\mathrm{r}=.071, p=9.40 \times 10^{-22}\right)$, but a non-significant genetic correlation between them $(\rho=.133$, $p=.053$, null hypothesis $=$ different to 0) (Table S6). After adjusting the AI for TBV using linear regression, the SNPbased heritability was virtually unchanged (for example a heritability of .14 using the GCTA method, the same as for the unadjusted AI; Fig. S7). L and R measures dropped somewhat in heritability after adjusting for TBV, although the 95\% confidence intervals overlapped with the unadjusted measures (Fig. S7). We did not adjust for TBV in the main genetic analysis of the PT AI, as we wished to capture all genetic influences on the PT AI, regardless of whether they might be shared with other aspects of brain anatomy. It is also not advisable in genetic analyses to adjust for covariates that are themselves heritable, as this can induce bias (Aschard, Vilhjalmsson, Joshi, Price, \& Kraft, 2015).

\subsection{Genome wide association analysis of PT measures}

Two loci on different chromosomes were significantly associated with the PT AI at a threshold $p<5 \times 10^{-8}$ (Table 1):

The most significant association with the PT AI was on chromosome 10p14 (rs41298373, $p=2.01 \times 10^{-15}$, Fig. 2a,S8). The minor A allele of $\mathrm{rs} 41298373(\mathrm{MAF}=.10)$ was associated with an increased leftward asymmetry of PT volume $(\mathrm{T}=6.18$, Fig. S9; Table S7). In fact this SNP was associated only with left-sided PT volume $\left(p=6.48 \times 10^{-10}\right)$ and not with right-sided PT volume $(p=.13)$ (Fig. S10, Table S7). Post hoc analysis showed that the association of rs41298373 with the PT AI was similar in males (beta $=.023$, se $=.0047, p=7.76 \times 10^{-07}$ ) and females (beta $=.028$, se $=.0046, p=4.46 \times 10^{-10}$ ), and the sex ${ }^{*}$ rs41298373 interaction was not significant $(\mathrm{F}=.541, p=.462)$. There are no other SNPs within $500 \mathrm{~kb}$ of rs41298373 that have LD with it at $\mathrm{r}^{2}>.5$, which explains why this association signal appears relatively specific to rs41298373 (Fig. S11). Nonetheless rs41298373 was directly genotyped and was in Hardy-Weinberg equilibrium $(p=.81)$, so that this result appears reliable.

The second most significant association with the PT AI was on $2 \mathrm{q} 37, p=7.54 \times 10^{-10}$ for the lead SNP rs7420166. There were 6 SNPs at this locus that had $p$ values below $5 \times 10^{-8}$, all in high-LD with each other $\left(r^{2}>.7\right.$ with the lead SNP) (Fig. 2b). The effect of rs7420166 on PT asymmetry was driven by opposite effects on the left PT (T=3.70, $\left.p=2 \times 10^{-4}\right)$ and the right $\mathrm{PT}(\mathrm{T}=-1.95, p=.051)$, with the minor allele $\mathrm{A}$ $(\mathrm{MAF}=.256)$ resulting in increased leftward asymmetry (Table S7). Post hoc analysis showed that the association of rs7420166 with the PT AI was similar in males (beta $=.016$, se $=.0034, p=4.12 \times 10^{-6}$ ) and females (beta $=.0129$, se $=.0031, p=4.47 \mathrm{e}-05)$, and the sex ${ }^{*}$ rs41298373 interaction was not significant $(\mathrm{F}=1.58, p=.209)$.

Neither of the lead SNPs rs41298373 and rs7420166, nor proxy SNPs in LD $\mathrm{r}^{2}>.8$ with them, were included in the previously published GWAS meta-analysis of PT asymmetry in roughly 3,000 subjects (Guadalupe et al. , 2015) (see Introduction).

No other loci surpassed the threshold $p<5 \times 10^{-8}$ in the GWAS of left PT, while a locus on chromosome 16q24.1 (lead SNP rs12932673, MAF $=.189$, minor allele $=$ G) just surpassed this threshold in the GWAS of the right PT volume $(\mathrm{T}=-5.59$, $p=2.315 \times 10^{-8}$, Fig. S10, Table S7). This SNP was also associated with the left PT volume to a lesser extent than the right $\left(\mathrm{T}=-2.76, p=5.845 \times 10^{-3}\right)$ such that the association with the PT AI was not even nominally significant ( $\mathrm{T}=1.94, p=.052)$.

None of the loci mentioned here showed association with TBV (all $p>$.1; Table S8), and the pattern of results for these loci was unchanged when the AI, L or R measures were adjusted for TBV (Table S8).

We checked the four SNPs that had pointwise association $p$ values below $1 \times 10^{-6}$ in the GWAS by Guadalupe et al. (2015) (none of which were significant at a genome-wide adjusted level in that study), but these SNPs were not associated with $\mathrm{PT}$ asymmetry in the present study (all uncorrected $p>.5$ ).

\subsection{Further characterisation of loci associated with PT asymmetry}

Rs41298373 on 10p14 is an exonic nonsynonymous SNP within exon 9 of ITIH5 (Inter-Alpha-Trypsin Inhibitor Heavy Chain Family Member 5) (transcript = ENST00000397145). The minor allele results in a substitution from alanine to valine (p.A376V), within the von Willebrand factor type A (VWA) domain of the protein (pfam = PF13768). The SNP is predicted to affect protein function by several bioinformatic tools (Polyphen = probably damaging, SIFT = deleterious, CADD $=27.9$ ). The Genomic Evolutionary Rate Profiling (GERP) estimate for this genomic position is high (5.23), reflecting that the ancestral allele $G$ is conserved across mammals. $G$ is also the allele at this site in the reference genomes of archaic hominins (two Neanderthals and one Denisovan) (Green et al., 2010; Meyer et al., 2012). The minor allele A has a frequency around $10 \%$ in most human populations, but is lower in African (2\%) and East-Asian (.7\%) groups according to the gnomAD database (Lek et al., 2016). 

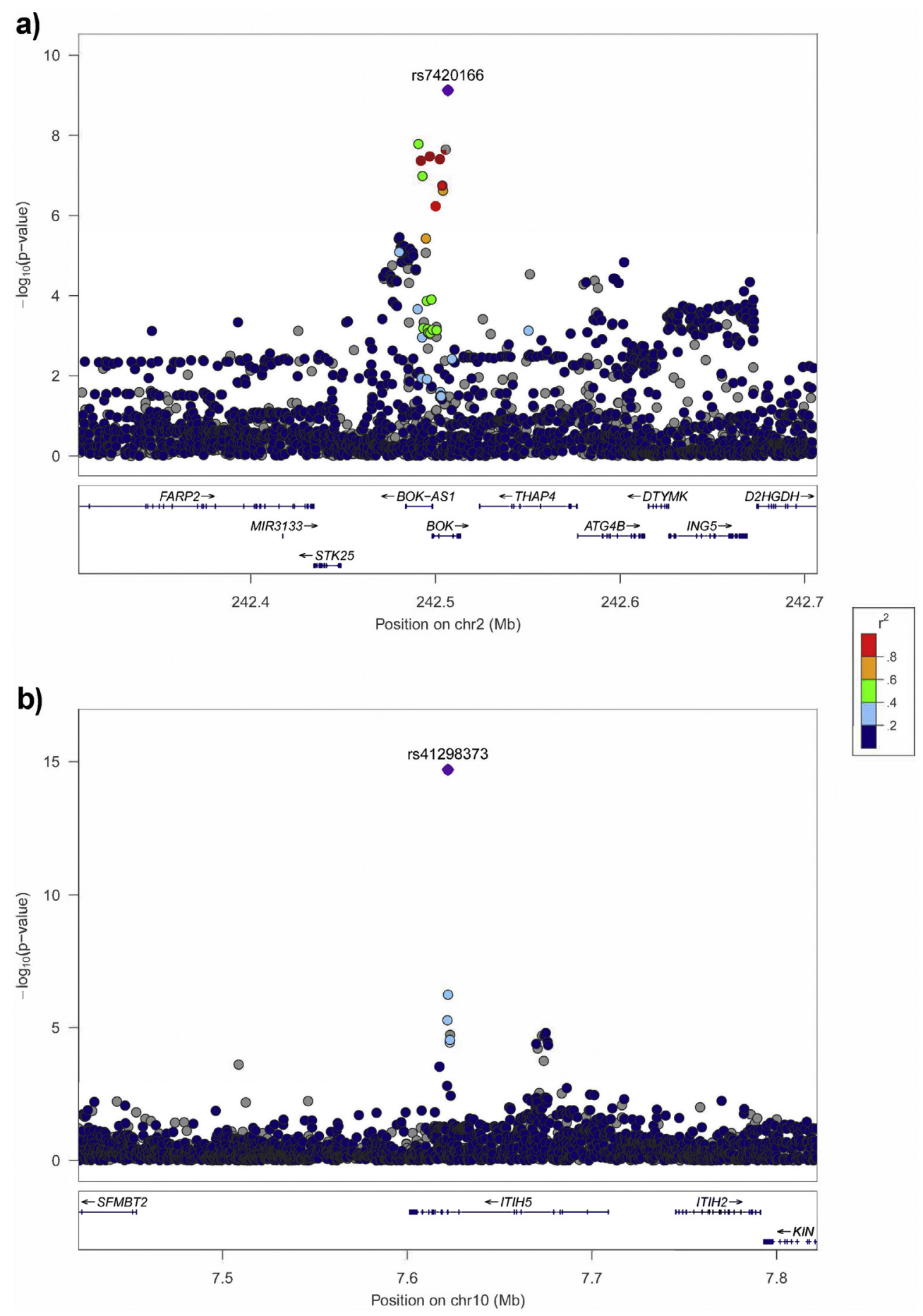

Fig. 2 - Regional association plot of part of (a) chr2q37 and (b) chr10p14, with PT volume asymmetry. The lead SNP (most significantly associated) is highlighted in violet, and the rest of the SNPs are coloured on the basis of their LD with the lead SNP (LD r ${ }^{2}$, within the 1 KG EUR population).

ITIH5 encodes a heavy chain component of one of the inter-alpha-trypsin inhibitor (ITI) family members (Himmelfarb et al., 2004). The ITI family contains multiple proteins (ITIP) that are made up of a light chain (bikunin) and a variable number of heavy chains (ITIH1-ITIH6), and are plasma serine protease inhibitors. They are involved in extracellular matrix stabilization and in prevention of tumor metastasis. ITIH5 is a known tumor suppressor gene, and it has been shown to be downregulated in breast cancer (Himmelfarb et al., 2004).

The expression pattern of ITIH5 is broad, being most highly expressed in adipose, artery, colon and mammary tissue (Consortium G., 2015). It also shows moderate expression in the brain throughout development (mean fragments per kilobase of exon per million reads mapped (FPKM) across all timepoints and regions in Brainspan $=2.86$, range: $.35-24.67$ 
(Miller et al., 2014); GTEx range of median Transcripts per million (TPM) across brain regions: 3.8-9.9), with a possible peak around the time of birth Fig. S12, but with no particular spatial patterns discernible (BRAINEAC, GTEx, AllenBrainAtlas) (Ramasamy et al., 2014; Consortium G., 2015; Hawrylycz et al., 2012). In the Allen data from adult post mortem brain, the expression pattern within the PT did not reveal significant hemispheric differences, although there was a non-significant trend toward lower expression on the right $\left[\beta=-.2824\right.$, se $=.1995 ;$ LRT $\left.\chi^{2}(1)=2.26, p=.13\right]$ (Fig. S13). Single-cell RNA sequencing data from the adult human Middle Temporal Gyrus (MTG), as well as brain cell-type transcriptome profiles of adult human (Zhang et al., 2016) and mouse (Zhang et al., 2014) brain, showed that ITIH5 is most highly expressed in the endothelial cells (EC) of the brain (Table S9; Fig. S14). This is consistent with its putative role as a brain-blood barrier marker (Daneman et al., 2010). In the mouse, Itih5 is one of the 50 most enriched transcripts in brain endothelial cells compared to liver endothelial (3124.8 fold enrichment), or lung endothelial cells (213.3) (Daneman et al., 2010). In situ hybridisation showed that this gene is expressed sporadically and specifically in larger vessels but not in smaller vessels (Daneman et al., 2010). A more recent characterization of the mouse CNS endothelial cell transcriptome showed Itih5 is enriched only in adult brain ECs (not in P7 brain ECs, nor in other tissue ECs) (Sabbagh et al., 2018).

As regards the locus on 2q37, rs7420166 is within the third intron of BOK (BCL2-Related ovarian killer) which encodes a member of the BCL2 family, known as essential regulators of the mitochondrial apoptosis pathway (Gross \& Katz, 2017; Haschka \& Villunger, 2017). The locus also encompasses BOK-AS1 (BOK antisense RNA 1). In peripheral blood tissue (Westra et al., 2013; Zhernakova et al., 2017), rs7420166 and additional SNPs within the associated locus act as eQTLs for BOK and 9 other genes in the region (FARP2, SEPT2, DTYMK, ING5, ATG4B, D2HGDH, AC005104.3, AC114730.7, AC114730.11) $(F D R<.05)$. This locus also affects the expression of BOK and DTYMK in adult cortex (unadjusted $p<.005$ ) (GO Consortium, 2015; Ng et al., 2017).

BOK is highly expressed in the central nervous system and multiple brain regions (Table S9) and has been implicated during neuronal apoptosis in mice (D'Orsi et al., 2016). BOK is highly expressed in endothelial cells of the mouse (Table S9), but low expression was detected across human brain cell types (Table S9, Fig. S14). In the Allen data from adult post mortem brain, the expression pattern within the PT did not reveal significant hemispheric differences (Fig. S13). Developmentally, BOK increases in expression levels in the human brain from embryo to mid gestation, and then remains at a steady level into adulthood (Fig. S12).

Rs7420166 is located $119 \mathrm{~kb}$ upstream of DTYMK. This gene encodes for an enzyme that catalyzes the phosphorylation of deoxythymidine monophosphate (dTMP) in the deoxythymidine triphosphate (dTTP) synthesis pathway, for DNA synthesis (Huang et al., 1994). It is most expressed in transformed fibroblasts and lymphocytes, but also present in the brain (most highly in the cerebellum). The expression in the left PT was significantly higher than in the right, in the Allen post mortem brain data $\left[\chi^{2}(1)=7.18, p=.007376\right]$ (Fig. S13). Developmentally, the expression of DTYMK decreases with age, with the highest expression levels at early prenatal timepoints (Fig. S12).

\subsection{Testing for broader effects of rs41298373 and rs7420166 on brain asymmetry}

Analysis of voxel-wise grey matter volume asymmetry (Fig. 3) showed that the effect of rs41298373 was strongest in a cluster (311 voxels, $\mathrm{Zmax}=7.66, \mathrm{MNI}=-40,-34,12$ ) overlapping with the PT and the parietal operculum, as defined in the Harvard-Oxford atlas (Fig. 3a). Two additional clusters had effects in the same direction (minor allele A associated with stronger leftward asymmetry): superior temporal gyrus (160 voxels, $\mathrm{Zmax}=4.49, \mathrm{MNI}=-62,-4,4)$ and temporal pole $(65$ voxels, $\mathrm{Zmax}=4.03$, MNI $=-58,10,-18$ ) (Fig. 3a). There were also two clusters showing an opposite pattern, i.e., more rightward grey matter volume asymmetry associated with the minor allele of rs41298373, in the parahippocampal gyrus (424 voxels, $\mathrm{Zmax}=5.45, \mathrm{MNI}=-28,-14,-30)$ and the posterior part of the superior temporal sulcus (112 voxels, $Z \max =4.3$, MNI $=-46,-44,4)$ (Fig. 3).

The effect of rs7420166 on grey matter volume asymmetry was largely confined to a single cluster within the PT (Fig. 3b, 111 voxels, $Z \max =4.22, \mathrm{MNI}=-40,-32,14$ ) (minor allele A associated with stronger leftward asymmetry) (Fig. 3b).

In surface-based analysis, there was no significant association of vertex-wise cortical thickness asymmetry with either rs41298373 or rs7420166. Vertex-wise analysis of cortical surface area left-right differences with rs41298373 revealed that the minor allelle $A$ is associated with a more leftward surface area asymmetry in a large cluster of $6380 \mathrm{~mm}^{2}$, overlapping with the lower part of the pre- and post-central gyri, the supra-marginal gyrus, the internal part of the superior temporal gyrus covering the PT, Heschl's gyrus and the posterior part of the insula, and extending to the temporal pole (Fig. 4). We also identified a second smaller cluster $\left(2217 \mathrm{~mm}^{2}\right)$ located in the rostral middle frontal gyrus and showing the opposite pattern (i.e., a more rightward surface area asymmetry associated with the minor allele of rs41298373) (Fig. 4a). As for rs7420166, we found that the minor allelle is associated with a more leftward cortical surface area asymmetry in a restricted cluster of $2205 \mathrm{~mm}^{2}$ overlapping the lower part of the postcentral gyrus, the more anterior part of the supra-marginal gyrus, the PT and the posterior part of Heschl's gyrus (Fig. 4b).

\subsection{Gene set enrichment analyses}

In gene set enrichment analysis of the GWAS results for PT AI, no gene sets were significantly enriched for association signals after correction for 5,875 tested GO sets.

Since a previous GWAS of PT asymmetry in roughly 3,000 subjects had reported significant enrichment of association in two steroid-related gene sets (Guadalupe et al., 2015), we also looked up the MAGMA enrichment results for these candidate sets as targeted hypotheses, but found no enrichment of association within 'steroid hormone receptor activity' (unadjusted $p=.68$ ) nor 'steroid metabolic process' (unadjusted $p=.42$ ). We also applied the same enrichment analysis software (Lee, OD; ushlaine, Thomas, \& Purcell, 2012) for testing these two gene sets as had been used in the study by 
a)

$$
\text { rs41298373 }
$$

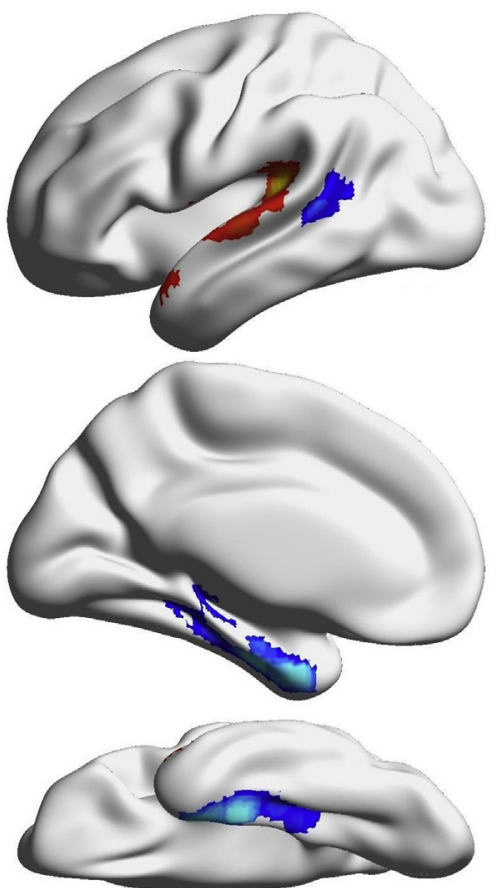

b) rs7420166

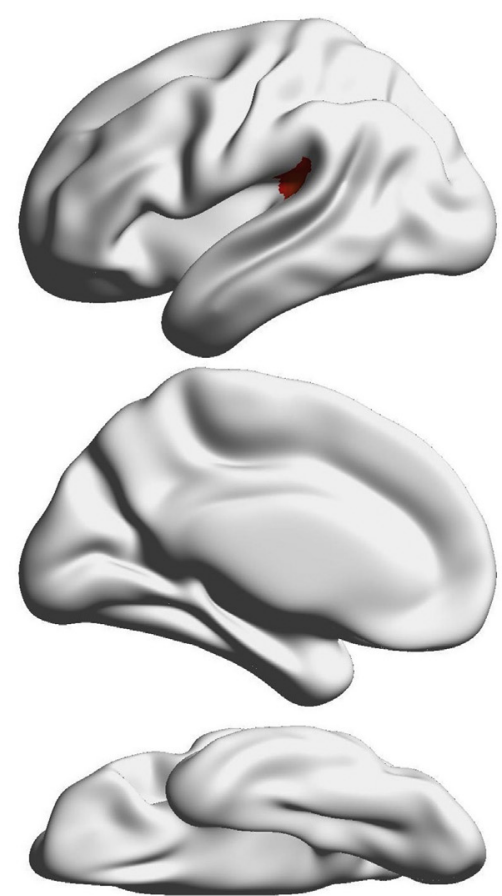

Effect of minor allele



Fig. 3 - Results of the grey matter VBM AI analysis of (a) rs7420166 and (b) rs41298373. Z-scores are thresholded at $|Z|>3$, cluster size $>\mathbf{4 0}$ voxels, and mapped onto a left-hemisphere surface model for visualization purposes. The direction and strength of the association is indicated by colour (red to yellow = minor allele associated with more leftward asymmetry, blue $=$ minor allele associated with more rightward asymmetry).

a)

$$
\text { rs41298373 }
$$
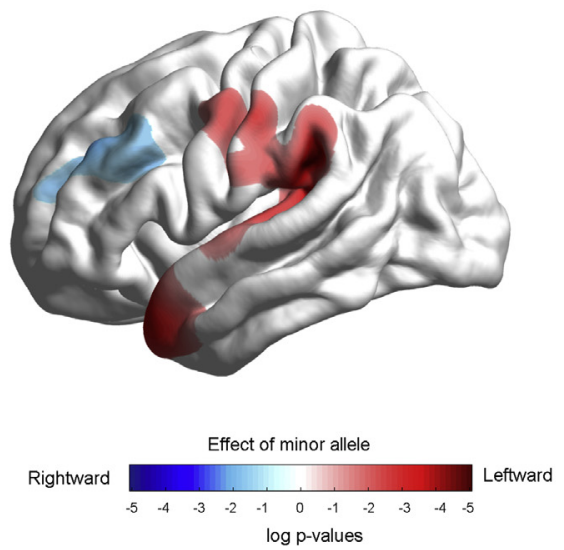

b)
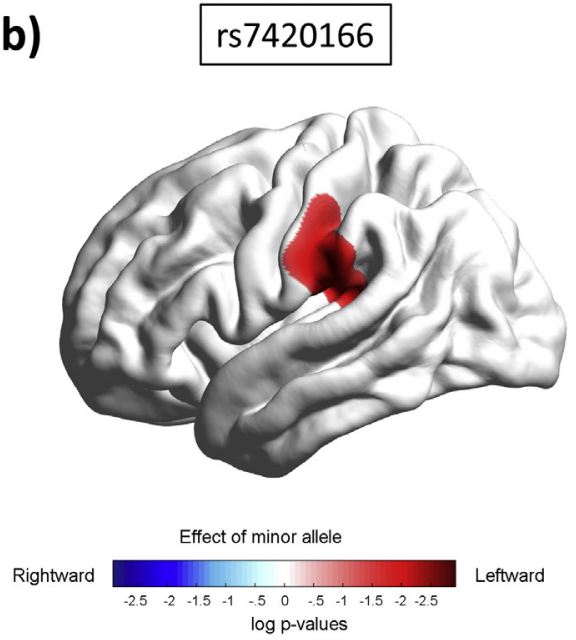

Fig. 4 - Association analysis of (a) rs41298373 and (b) rs7420166 with vertex-wise cortical surface area left-right differences. The direction and strength of the association is indicated by colour (red = minor allele associated with more leftward asymmetry, blue $=$ minor allele associated with more rightward asymmetry). Monte-Carlo simulation corrected log-values $p<.05$ are shown. 
Guadalupe et al. (2015), but again saw no evidence for enrichment (both $p>.5$ ).

\subsection{Little evidence for genetic links between PT asymmetry and SCZ, ASD, ADHD, intelligence or educational attainment}

Using publicly available GWAS summary statistics from large scale studies of SCZ, ASD, ADHD, intelligence and EA (Table S5), we found no significant genetic correlations of these traits with PT asymmetry, with a variety of different genetic models and methods (all $p>.05$, see Table S10 and Fig. S15). Considered separately, the left and right PT volumes were positively genetically correlated with intelligence and EA (Table S10). These genetic correlations were consistent across two out of three methods for calculating them (LDSC and the ldak model of SumHer, Table S10), but were no longer significant after adjusting the left and right PT volumes for TBV, so that these genetic correlations may have largely reflected a general relation of brain size to intelligence and EA. We had adequate power $(\beta>.80)$ to detect genetic correlations with PT asymmetry above roughly $.05-.15$, depending on the trait/ model, and as low as .025 with the left and right volumes owing to their higher heritabilities than the AI (see Fig. S16; Fig. S7 Table S3).

In addition, we checked whether either of the two genomewide significant associations with PT asymmetry which we had detected, i.e., the SNPs rs41298373 and rs7420166, or proxy SNPs for rs7420166, were also associated with any of SCZ, ASD, ADHD, intelligence or EA. There were only two nominally significant associations: between rs41298373 and intelligence $(p=.025)$ (Savage et al., 2018), and between three proxy SNPs in high LD with rs7420166 $\left(\mathrm{r}^{2}>.80\right)$ and EA (all $p<.05$, Table S11). Note that not all of the various GWAS summary statistics included data on these SNPs or their proxies for the purposes of this look-up (see Table S11).

\section{Discussion}

It has been a longstanding hypothesis that asymmetry of the PT is linked genetically to variation in human cognition and disorder susceptibility (Crow, 1993; Sommer, Ramsey, Kahn, Aleman, \& Bouma, 2001), given evidence for associations at the phenotypic level (see Introduction). Here we leveraged molecular genetic and neuroimaging data, which have only become available on a sufficiently large scale very recently, to investigate this question from a polygenic perspective. Overall, our analysis yielded novel insights into the genetic contributions to PT asymmetry, but did not support a clear role of these in cognitive variation and disorder susceptibility, at least that could be detected in current sample sizes.

\subsection{Genetics of PT asymmetry}

Within the UK Biobank dataset, we showed that asymmetry of the PT has a modest but significant SNP-based heritability, estimated at $14 \%$. In principle, a heritable component to PT asymmetry may arise because some genetic influences are quantitatively different on the left and right volumes of this structure, and we found evidence to support this, as the genetic correlation between left and right PT volumes was significantly less than 1 . However, PT asymmetry may itself be considered a primary brain phenotype, not merely arising from different effects on left and right. This conception would be consistent with the early appearance of PT asymmetry in development in utero, and its association with disorders when disrupted. Thus PT asymmetry may be analogous to the placement of the visceral organs on the left-right body axis (heart, lungs etc.), which arises due to a well-regulated genetic-developmental program in most people, but can be disrupted by various genetic mutations and environmental insults (Deng et al., 2015). In other words, there are probably some genetic influences on primary development of the brain's left-right axis, as well as other genetic influences that can have different effects on the left and right sides, which may come into play after the two sides have already been sent on different developmental trajectories. Asymmetry is also likely to be affected and maintained by interactions between the two hemispheres throughout development and adulthood. Studies in which gene expression has been contrasted between the left and right sides of the embryonic and foetal central nervous systems have provided support for a genetic developmental program underlying brain asymmetry (de Kovel et al., 2017; de Kovel, Lisgo, Fisher, \& Francks, 2018; Ocklenburg et al., 2017; Sun et al., 2005), although they could not specifically target the primitive PT itself.

A nonsynonymous variant within ITIH5 was significantly associated with PT asymmetry, for which the minor allele increased the left PT volume, but did not affect the right. VBM association analysis indicated that this variant affects asymmetry of the PT and the directly neighbouring parietal opercular cortex most strongly, but also extends to other temporal regions. Interestingly, the effect on asymmetry is in opposite directions in the PT and nearby middle temporal gyrus, suggesting that this variant affects the relative distribution of cortical tissue between these two left-hemisphere regions, and/or inter-hemispheric differences in the Sylvian fissure's position and ending. In vertex-wise, surface-based analysis using hemispheric co-registration, the effect is strongest in the PT area but also extends along the superior temporal surface area.

The non-synonymous variant in ITIH5 occurs at an evolutionarily conserved site, and the minor allele, which is the derived rather than ancestral allele, results in an amino acid change that is predicted to affect protein function and to be deleterious (i.e., selected against). Functional experiments will be needed to confirm whether the protein is impacted by this variant and how this might affect regional cortical development. From the perspective of language evolution, it is intriguing to note that a variant in the modern human population which leads to a larger left PT volume might also be selected against. However, any deleteriousness arising from this allele is not necessarily mediated through its effects on left PT volume or asymmetry. ITIH5 is also expressed in many other non-brain tissues, with an apparent role in stabilizing the extracellular matrix.

From various data sources we found that ITIH5 is expressed at moderate levels in the brain, in a mostly constant manner across regions and developmental stages, and with high 
expression in endothelial cells, consistent with a potential role as a blood-brain-barrier protein. There was a nonsignificant trend toward lower right-than left-side expression in the adult PT, in the Allen brain data, but the number of samples was too low to yield a conclusive result regarding expression levels of this gene. Overall, the available expression data for ITIH5 do not point to a clear explanation for the regional specificity of this gene-brain association, which may arise due to interactions of ITIH5 with other hemisphere- and region-specific factors.

Another insight into the genetics of PT asymmetry involved a locus on 2q37 which affects the expression level of BOK, a gene which has a role in neuronal apoptosis (D'Orsi et al., 2016), and also the kinase DTYMK at the same locus, which is involved in DNA synthesis and is expressed at a higher level on the left PT than the right, in post mortem adult tissue. The VBM association analysis indicated that this effect is specific to asymmetry of the PT region. However, vertex-wise analysis indicated an association with cortical surface area asymmetry that is somewhat more diffuse, covering a broader region of the internal part of the superior temporal gyrus, including the PT. This may be due to more accurate inter-hemispheric alignment in the surface-based analysis as compared to the voxel-based one. Again, interactions of this gene with other hemisphere and regionspecific factors may explain this restricted gene-brain association pattern.

Within the GWAS results for PT asymmetry based on over 18,000 subjects, there was no enrichment of association within the GO gene sets 'steroid hormone receptor activity' or 'steroid metabolic process'. This is in contrast to a previous GWAS of PT asymmetry in roughly 3,000 subjects, that reported significant enrichment of association in these two steroid-related gene sets ( $p$ values of .013 and .014 respectively, after adjustment for multiple testing). The current study and the previous study differ in a number of respects, which include the average age of participants, the precise method of PT volume measurement, sample size, genetic imputation panel, and method for gene-set enrichment analysis (see details of the study differences in Table S12). Overall, the present study is both larger and uses more up-to-date analytic approaches (Table S12). The present results in relation to steroid gene sets may therefore be taken to supersede the previous results, although the results of the previous study should also continue to be tested as available datasets expand. For example, it is possible that variation in PT asymmetry in younger adults (as included in the study by Guadalupe et al. (2015)) is more affected by steroid hormone influences than in older adults (as in the UK Biobank).

\subsection{Relation to SCZ, ASD, ADHD, intelligence, $E A$, and handedness}

In terms of phenotypic associations, PT asymmetry was not associated with handedness or fluid intelligence, but was weakly associated with educational years. Fluid intelligence and handedness were associated with PT volume bilaterally, but not asymmetrically. Effect sizes were very small after adjusting for total brain volume (partial correlations <.03), so that it is not possible to envisage PT volumes as biomarkers for these cognitive or behavioural traits. It would be interesting in future studies to map bilateral regional brain associations with cognitive and behavioural tasks across the whole cortex.

We had $80 \%$ statistical power to detect genetic correlations with PT asymmetry larger than roughly .05-.10 for SCZ, intelligence and EA, or .15 for ASD and ADHD, given the sample sizes used to derive the GWAS summary statistics for these traits, and their SNP-based heritabilities. Despite this, no significant genetic correlations were detected between PT asymmetry and these traits or disorders. One possibility is that previous studies which have shown phenotypic associations between PT asymmetry and SCZ and ASD in particular, have reported false positive findings based on limited sample sizes and/or publication bias in meta-analysis. In general there is an urgent need for the field to increase sample sizes in brain-disorder association studies. As regards ADHD, intelligence and EA, we are not aware of studies which have reported direct phenotypic correlations with PT asymmetry, but such reports have been made for dyslexia, while reading ability shares genetic contributions with ADHD, intelligence and EA (see Introduction). We await GWAS studies of reading measures in more than 10,000 subjects, to perform future genetic correlation analyses with PT asymmetry.

It is also possible that phenotypic associations between PT asymmetry and cognitive/psychiatric disorders may not be genetically driven, but caused by environmental factors. In line with this, a previous study also found no genetic overlap between SCZ and eight brain volume measures (mainly subcortical, and not with reference to asymmetry), despite robust phenotypic associations having been reported of these volumes with SCZ (Franke et al., 2016).

A third possibility is that genetic correlations do exist between PT asymmetry and some of SCZ, ASD, ADHD, intelligence, EA, but that the correlations are too low to have been detected with the present sample sizes. The GWAS for PT asymmetry in the present study was based on over 18,000 subjects, while the disorder GWAS were based on over 20,000 cases each, the intelligence GWAS over 250,000 individuals, and the EA GWAS over 750,000 individuals. As sample sizes increase further in the coming years, it may still be possible to detect genetic correlations of PT asymmetry with some of these traits, of the order of .05 or lower. Regardless, we can already conclude that PT asymmetry probably does not have a substantial genetic correlation with any of these traits.

We also explored the genetic correlations of the left and right PT volumes separately with SCZ, ASD, ADHD, intelligence, EA. In this case, as the left and right volume measures had higher heritabilities than the AI, we had $80 \%$ power to detect genetic correlations as low as .05 . We found that intelligence and EA had positive genetic correlations with both left and right PT volumes to similar extents. However, after adjusting the left and right PT volumes for TBV, the genetic correlations with intelligence and EA were largely diminished and non-significant. A previous study reported that average total cortical surface area is genetically correlated with EA $\left(\mathrm{rg}=.213, p=9.47 \times 10^{-13}\right)$ and general cognitive function $\left(\mathrm{rg}=.2221, p=3.94 \times 10^{-8}\right)$ (Grasby et al., 2018). Hence, the genetic correlations of left and right $\mathrm{PT}$ volumes with 
intelligence and EA, which we detected in the present study, are likely to reflect global effects of brain size, rather than region-specific effects, and are not linked to laterality.

Regarding the two individual loci which were significantly associated with PT asymmetry in the GWAS for this trait, we did not find evidence that they were associated with SCZ, ASD, or ADHD. The non-synonymous SNP in ITIH5 was nominally associated with intelligence (unadjusted $p=.02$ ) with a very small effect size (standard beta $=.011$ ). Thus, it is possible that this locus does impact intelligence, although a $p$ value of .02 based on a study of 269,867 individuals means that this could only be clarified in a much larger, future dataset. Rs7420166 was not available in the intelligence and EA GWASes, but three SNPs in high LD with it $\left(\mathrm{r}^{2}>\right.$.8: $\mathrm{rs} 114606940, \mathrm{rs} 73123528$, rs77278412) were nominally associated with EA (unadjusted Ps <.05). Again, these effects (beta $=.004$ ) were very small, detectable only in a dataset of 766,345 individuals.

As we found no phenotypic correlation of PT asymmetry with handedness, we did not pursue possible genetic correlation between these traits. A lack of correlation between PT asymmetry and handedness has also been reported by five previous, recent studies (Guadalupe et al., 2014, 2015; Maingault, Tzourio-Mazoyer, Mazoyer, \& Crivello, 2016; Tzourio-Mazoyer et al., 2010; Tzourio-Mazoyer \& Mazoyer, 2017). This is a further testament to brain asymmetry being a complex, multivariate trait (de Kovel, Lisgo, Fisher, \& Francks, 2018; Francks, 2015; Tzourio-Mazoyer et al., 2019; Vingerhoets, 2019), with potentially distinct developmental bases for its different aspects.

\subsection{Limitations}

Limitations with regard to sample size, statistical power, and the lack of large scale GWAS for dyslexia have already been discussed above. As regards dyslexia, the phenotypic association with PT asymmetry has been reported to be limited to boys (see Introduction), which means that further sex-limited genetic correlation analysis may be warranted. For the present study, the brain imaging data in the UK Biobank would be reduced to groups of roughly 8,500-9,000 if split by sex, which is too low to perform well powered genetic correlation analysis with other datasets by use of summary statistics, for a trait such as PT asymmetry with only $14 \%$ heritability. Also, the GWAS summary statistics for SCZ, ASD, intelligence and EA were not available split by sex. Although sex-stratified GWAS summary statistics were available for ADHD, the power to detect genetic correlation of PT asymmetry with this disorder would not have been adequate in sex-split analysis. In this study we controlled for sex as a covariate, and also found no evidence for a sex-specific effect of the top loci associated with PT asymmetry.

The Harvard-Oxford atlas, which we used to define the PT, was derived from manual segmentations of sets of reference brain images (Destrieux, Fischl, Dale, \& Halgren, 2010; Goldstein et al., 1999, 2007). It therefore contained asymmetrical definitions for regions that showed asymmetry in the reference dataset (including the PT). Accordingly, the measurement of average PT asymmetry in the UK Biobank dataset would partly reflect left-right differences present in the atlas. Nonetheless, the use of a 'real-world' asymmetrical atlas, rather than an artificially created symmetrical atlas, was appropriate for our primary purpose which was the GWAS of PT asymmetry in as large a sample as possible. This is because regional identification was likely to be more accurate for structures that were asymmetrical both in the atlas and, on average, in the UK Biobank dataset, and the GWAS analysis is based on comparing relative levels of asymmetry between genotype groups, rather than measuring absolute asymmetry levels. In addition, we followed up the most significant SNP associations with PT asymmetry that arose in the GWAS, by performing brainwide grey matter asymmetry VBM association analysis, this time based on a symmetrized template and no regional atlas definitions, which produced results that were highly consistent with the GWAS analysis. We further investigated vertex-wise effects without regard to any regional atlas definitions, and using a co-registration approach to improve interhemispheric correspondence.

\subsection{Future perspectives}

This study provided various novel insights into the genetic contributions to PT asymmetry, but did not find evidence that these are substantially shared with cognitive variation or susceptibility to psychiatric disorders. Future studies based on even larger samples may be able to tease out very subtle genetic correlations.

Functional asymmetry is likely to relate to asymmetries at the molecular and microanatomical levels (Chance, 2014; Karlebach \& Francks, 2015; Ocklenburg et al., 2018; Galuske, Schlote, Bratzke, \& Singer, 2000), so that studies beyond macroscopic anatomy are warranted, for example in terms of gene expression asymmetries, neurite architecture, and the abundances of neuronal subclasses.

Comparative studies have shown that chimpanzees have a leftward asymmetry of the Sylvian fissure and PT, while several old world monkey species do not (Yeni-Komshian \& Benson, 1976; Lyn et al., 2011). A recent study reported the first population-level leftward asymmetry of the PT in a nonhominin primate (olive baboon monkeys), dating the origin of this cerebral specialization as early as 30-40 million years ago (Marie et al., 2018). Therefore PT asymmetry is not a uniquely human feature. The involvement of the PT in language processing, which usually shows left-side dominance, probably arose through adaption of an already-lateralized brain infrastructure. Nonetheless, future studies may interrogate the genetics of PT asymmetry from the perspective of genomic conservation and signals of selection.

\section{Open practices}

The study in this article earned an Open Data - Protected Access badge for transparent practices. Data for the study is available at https://www.ukbiobank.ac.uk/using-the-resource/. The code associated to this study is publicly available at https://github. $\mathrm{com} /$ amaiacc/GeneticsPlanumTemporaleAsymmetry. GWAS summary statistics are available from the NHGRI-EBI GWAS Catalog https://www.ebi.ac.uk/gwas/downloads/summary-stat istics. 


\section{Declaration of Competing Interest}

The authors declare that they have no conflict of interest.

\section{CRediT authorship contribution statement}

Amaia Carrion-Castillo: Conceptualization, Methodology, Software, Visualization, Writing - original draft, Writing - review \& editing. Antonietta Pepe: Software, Writing - original draft, Visualization. Xiang-Zhen Kong: Software, Visualization. Simon E. Fisher: Conceptualization, Funding acquisition, Writing - review \& editing. Bernard Mazoyer: Conceptualization, Supervision, Funding acquisition, Writing - review \& editing. Nathalie Tzourio-Mazoyer: Conceptualization, Supervision, Funding acquisition, Writing - review \& editing. Fabrice Crivello: Conceptualization, Supervision, Writing original draft, Funding acquisition, Writing - review \& editing. Clyde Francks: Conceptualization, Funding acquisition, Supervision, Writing - original draft, Writing - review \& editing.

\section{Acknowledgements}

This research was conducted using the UK Biobank Resource under Application Number 16066, with Clyde Francks as the principal applicant. AC-C was funded by a grant to CF from the Netherlands Organisation for Scientific Research (NWO) (05415-101), and B.M, N.T.M., A.P., and F.C by a grant from the French National Research Agency (ANR, grant No. 15-HBPR0001-03), as part of the FLAG-ERA consortium project 'MULTILATERAL', a Partner Project to the European Union's Flagship Human Brain Project. Additional support was from the Max Planck Society (Germany).

\section{Supplementary data}

Supplementary data to this article can be found online at https://doi.org/10.1016/j.cortex.2019.11.006.

\section{R E F E R E N C E S}

Altarelli, I., Leroy, F., Monzalvo, K., Fluss, J., Billard, C., DehaeneLambertz, G., \& Ramus, F. (2014 Dec). Planum temporale asymmetry in developmental dyslexia: Revisiting an old question. Human Brain Mapping, 35(12), 5717-5735.

Aschard, H., Vilhjalmsson, B. J., Joshi, A. D., Price, A. L., \& Kraft, P. (2015 Feb). Adjusting for heritable covariates can bias effect estimates in genome-wide association studies. American Journal of Human Genetics, 96(2), 329-339.

Ashburner, M., Ball, C. A., Blake, J. A., Botstein, D., Butler, H., Cherry, J. M., et al. (2000 May). Gene ontology: Tool for the unification of biology. The gene Ontology consortium. Nature Genetics, 25(1), 25-29.

Bryden, M. P., McManus, I. C., \& Bulman-Fleming, M. B. (1994 Nov). Evaluating the empirical support for the GeschwindBehan-Galaburda model of cerebral lateralization. Brain and Cognition, 26, 103-167.
Bulik-Sullivan, B. K., Loh, P. R., Finucane, H. K., Ripke, S., Yang, J., Patterson, N., et al. (2015 Mar). LD Score regression distinguishes confounding from polygenicity in genome-wide association studies. Nature Genetics, 47(3), 291-295.

Bycroft, C., Freeman, C., Petkova, D., Band, G., Elliott, L. T. Sharp, K., et al. (2018 Oct). The UK Biobank resource with deep phenotyping and genomic data. Nature, 562(7726), 203-209.

Chance, S. A. (2014). The cortical microstructural basis of lateralized cognition: A review. Frontiers in Psychology, 5, 820.

Chi, J. G., Dooling, E. C., \& Gilles, F. H. (1977 Jun). Left-right asymmetries of the temporal speech areas of the human fetus. Archives in Neurology, 34(6), 346-348.

Crow, T. (1993). Schizophrenia as an anomaly of cerebral asymmetry. In K. Maurer (Ed.), Imaging of the brain in psychiatry and related fields. Berlin Heidelberg: Springer-Verlag.

Crow, T., Ball, J., Bloom, S., \& Al, E. (1989). Schizophrenia as an anomaly of development of cerebral asymmetry: A postmortem study and a proposal concerning the genetic basis of the disease. Archives of General Psychiatry, 46(12), 1145-1150.

D'Orsi, B., Engel, T., Pfeiffer, S., Nandi, S., Kaufmann, T., Henshall, D. C., et al. (2016, Apr). Bok is not pro-apoptotic but suppresses poly ADP-ribose polymerase-dependent cell death pathways and protects against excitotoxic and seizureinduced neuronal injury. The Journal of Neuroscience, 36(16), 4564-4578.

Dale, A., Fischl, B., \& Sereno, M. I. (1999). Cortical surface-based analysis: I. Segmentation and surface reconstruction. Neuroimage, 9(2), 179-194.

Daneman, R., Zhou, L., Agalliu, D., Cahoy, J. D., Kaushal, A., \& Barres, B. A. (2010, Oct). The mouse blood-brain barrier transcriptome: A new resource for understanding the development and function of brain endothelial cells. PLoS One, 5(10), e13741.

de Kovel, C. G., Lisgo, S. N., Fisher, S. E., \& Francks, C. (2018, Sep). Subtle left-right asymmetry of gene expression profiles in embryonic and foetal human brains. Scientific Reports, 8(1), 12606.

de Kovel, C. G., Lisgo, S., Karlebach, G., Ju, J., Cheng, G., Fisher, S. E., et al. (2017, Aug). Left-right asymmetry of maturation rates in human embryonic neural development. Biological Psychiatry, 82(3), 204-212.

de Leeuw, C. A., Mooij, J. M., Heskes, T., \& Posthuma, D. (2015, Apr). MAGMA: Generalized gene-set analysis of GWAS data. PLoS Computational Biology, 11(4), e1004219.

Demontis, D., Walters, R. K., Martin, J., Mattheisen, M., Als, T. D., Agerbo, E., et al. (2019, Jan). Discovery of the first genome-wide significant risk loci for attention deficit/hyperactivity disorder. Nature Genetics, 51(1), 63-75. https://doi.org/10.1038/s41588018-0269-7.

Deng, H., Xia, H., \& Deng, S. (2015, Jan). Genetic basis of human left-right asymmetry disorders. Expert Reviews in Molecular Medicine, 16, e19.

Desikan, R. S., Ségonne, F., Fischl, B., Quinn, B. T., Dickerson, B. C., Blacker, D., et al. (2006). An automated labeling system for subdividing the human cerebral cortex on MRI scans into gyral based regions of interest. Neuroimage, 31(3), 968-980. Retrieved from http://www.sciencedirect.com/science/article/B6WNP4JFHF4P-1/2/0ec667d4c17eafb0a7c52fa3fd5aef1c.

Destrieux, C., Fischl, B., Dale, A., \& Halgren, E. (2010, Oct). Automatic parcellation of human cortical gyri and sulci using standard anatomical nomenclature. Neuroimage, 53(1), 1-15.

Douaud, G., Smith, S., Jenkinson, M., Behrens, T., JohansenBerg, H., Vickers, J., et al. (2007, Sep). Anatomically related grey and white matter abnormalities in adolescent-onset schizophrenia. Brain, 130(Pt 9), 2375-2386.

Dronkers, N. F., Wilkins, D. P., Van Valin, R. D., Redfern, B. B., \& Jaeger, J. J. (2004). Lesion analysis of the brain areas involved in language comprehension. Cognition, 92(1-2), 145-177. 
Dubois, J., Benders, M., Lazeyras, F., Borradori-Tolsa, C., Leuchter, R. H., Mangin, J. F., et al. (2010, Aug). Structural asymmetries of perisylvian regions in the preterm newborn. Neuroimage, 52(1), 32-42.

Fischl, B., Sereno, M. I., \& Dale, A. (1999). Cortical surface-based analysis: II: Inflation, flattening, and a surface-based coordinate system. Neuroimage, 9(2), 195-207.

Foundas, A. L., Leonard, C. M., Gilmore, R., Fennell, E., \& Heilman, K. M. (1994, Oct). Planum temporale asymmetry and language dominance. Neuropsychologia, 32(10), 1225-1231.

Francks, C. (2015, Nov). Exploring human brain lateralization with molecular genetics and genomics. Annals of the New York Academy of Sciences, 1359, 1-13.

Franke, B., Stein, J. L., Ripke, S., Anttila, V., Hibar, D. P., van Hulzen, K. J., et al. (2016, Mar). Genetic influences on schizophrenia and subcortical brain volumes: Large-scale proof of concept. Nature Neuroscience, 19(3), 420-431.

Galaburda, A. M., Corsiglia, J., Rosen, G. D., \& Sherman, G. F. (1987). Planum temporale asymmetry, reappraisal since Geschwind and Levitsky. Neuropsychologia, 25(6), 853-868.

Galaburda, A. M., Sherman, G. F., Rosen, G. D., Aboitiz, F., \& Geschwind, N. (1985, Aug). Developmental dyslexia: Four consecutive patients with cortical anomalies. Annals of Neurology, 18(2), 222-233.

Galuske, R. A., Schlote, W., Bratzke, H., \& Singer, W. (2000, Sep). Interhemispheric asymmetries of the modular structure in human temporal cortex. Science, 289, 1946-1949.

Geschwind, N., \& Galaburda, A. (1985, june). Cerebral lateralization. Biological mechanisms, associations, and pathology: I. A hypothesis and a program for research. Archives of Neurology, 42, 428-459.

Geschwind, N., \& Levitsky, W. (1968, Jul). Human brain: Left-right asymmetries in temporal speech region. Science, 161(3837), 186-187.

GO Consortium. (2015). Human genomics. The genotype-tissue expression (GTEx) pilot analysis: Multitissue gene regulation in humans. Science, 348(6235), 648-660.

GO Consortium. (2017). Expansion of the gene ontology knowledgebase and resources. Nucleic Acids Research, 45(D1), D331-D338.

Goldstein, J. M., Goodman, J. M., Seidman, L. J., Kennedy, D. N., Makris, N., Lee, H., et al. (1999, Jun). Cortical abnormalities in schizophrenia identified by structural magnetic resonance imaging. Archives in General Psychiatry, 56(6), 537-547.

Goldstein, J. M., Seidman, L. J., Makris, N., Ahern, T., O'Brien, L. M., Caviness, V. S., et al. (2007, Apr). Hypothalamic abnormalities in schizophrenia: Sex effects and genetic vulnerability. Biological Psychiatry, 61(8), 935-945.

Grasby, K. L., Jahanshad, N., Painter, J. N., Colodro-Conde, L., Bralten, J., Hibar, D. P., et al. (2018). The genetic architecture of the human cerebral cortex. bioRxiv. https://doi.org/10.1101/ 399402. Retrieved from https://www.biorxiv.org/content/ early/2018/09/09/399402.

Green, R. E., Krause, J., Briggs, A. W., Maricic, T., Stenzel, U., Kircher, M., et al. (2010, May). A draft sequence of the Neandertal genome. Science, 328(5979), 710-722.

Greve, D. N., Van der Haegen, L., Cai, Q., Stufflebeam, S., Sabuncu, M. R., Fischl, B., et al. (2013, Sep). A surface-based analysis of language lateralization and cortical asymmetry. Journal in Cognitive Neuroscience, 25(9), 1477-1492.

Gross, A., \& Katz, S. G. (2017,Aug). Non-apoptotic functions of BCL-2 family proteins. Cell Death and Differentiation, 24(8), 1348-1358.

Grove, J., Ripke, S., Als, T. D., Mattheisen, M., Walters, R., Won, H., et al. (2019, Mar). Identification of common genetic risk variants for autism spectrum disorder. Nature Genetics, 51(3), 431-444. https://doi.org/10.1038/s41588-019-0344-8.
Guadalupe, T., Willems, R. M., Zwiers, M. P., Arias Vasquez, A., Hoogman, M., Hagoort, P., et al. (2014). Differences in cerebral cortical anatomy of left- and right-handers. Frontiers in Psychology, 5, 261.

Guadalupe, T., Zwiers, M. P., Wittfeld, K., Teumer, A., Vasquez, A. A., Hoogman, M., et al. (2015, Jan). Asymmetry within and around the human planum temporale is sexually dimorphic and influenced by genes involved in steroid hormone receptor activity. Cortex, 62, 41-55.

Hasan, A., Kremer, L., Gruber, O., Schneider-Axmann, T., Guse, B., Reith, W., et al. (2011, Jul). Planum temporale asymmetry to the right hemisphere in first-episode schizophrenia. Psychiatry Research, 193(1), 56-59.

Haschka, M. D., \& Villunger, A. (2017, Mar). There is something about BOK we just don't get yet. The FEBS Journal, 284(5), 708-710.

Hawrylycz, M. J., Lein, E. S., Guillozet-Bongaarts, A. L., Shen, E. H., Ng, L., Miller, J. A., et al. (2012, Sep). An anatomically comprehensive atlas of the adult human brain transcriptome. Nature, 489(7416), 391-399.

Himmelfarb, M., Klopocki, E., Grube, S., Staub, E., Klaman, I., Hinzmann, B., et al. (2004, Feb). ITIH5, a novel member of the inter-alpha-trypsin inhibitor heavy chain family is downregulated in breast cancer. Cancer Letters, 204(1), 69-77.

Hirayasu, Y., McCarley, R. W., Salisbury, D. F., Tanaka, S., Kwon, J. S., Frumin, M., et al. (2000, Jul). Planum temporale and Heschl gyrus volume reduction in schizophrenia: A magnetic resonance imaging study of first-episode patients. Archives in General Psychiatry, 57(7), 692-699.

Hollier, L. P., Maybery, M. T., Keelan, J. A., Hickey, M., \& Whitehouse, A. J. (2014, Dec). Perinatal testosterone exposure and cerebral lateralisation in adult males: Evidence for the callosal hypothesis. Biological Psychology, 103, 48-53.

Huang, S. H., Tang, A., Drisco, B., Zhang, S. Q., Seeger, R., Li, C., et al. (1994, May). Human dTMP kinase: Gene expression and enzymatic activity coinciding with cell cycle progression and cell growth. DNA and Cell Biology, 13(5), 461-471.

Jakab, A., Meuwly, E., Feldmann, M., Rhein, M. V., Kottke, R., O'Gorman Tuura, R., et al. (2019, May). Left temporal plane growth predicts language development in newborns with congenital heart disease. Brain, 142(5), 1270-1281.

Jenkinson, M., Beckmann, C. F., Behrens, T. E., Woolrich, M. W., \& Smith, S. M. (2012, Aug). FSL. Neuroimage, 62(2), 782-790.

Karlebach, G., \& Francks, C. (2015, Jun). Lateralization of gene expression in human language cortex. Cortex, 67, 30-36.

Keller, S. S., Roberts, N., Garcia-Finana, M., Mohammadi, S., Ringelstein, E. B., Knecht, S., et al. (2011, Aug). Can the language-dominant hemisphere be predicted by brain anatomy? Journal in Cognitive Neuroscience, 23(8), 2013-2029.

Knaus, T. A., Kamps, J., Foundas, A. L., \& Tager-Flusberg, H. (2018, Oct). Atypical PT anatomy in children with autism spectrum disorder with expressive language deficits. Brain Imaging and Behavior, 12(5), 1419-1430.

Lee, J. J., Wedow, R., Okbay, A., Kong, E., Maghzian, O., Zacher, M., et al. (2018, Jul). Gene discovery and polygenic prediction from a genome-wide association study of educational attainment in 1.1 million individuals. Nature Genetics, 50(8), 1112-1121.

Lee, P. H., ODushlaine, C., Thomas, B., \& Purcell, S. M. (2012, Jul). Inrich: Interval-based enrichment analysis for genome-wide association studies. Bioinformatics, 28, 1797-1799.

Lek, M., Karczewski, K. J., Minikel, E. V., Samocha, K. E., Banks, E., Fennell, T., et al. (2016, Aug). Analysis of protein-coding genetic variation in 60,706 humans. Nature, 536(7616), 285-291.

Li, G., Nie, J., Wang, L., Shi, F., Lyall, A. E., Lin, W., et al. (2014, May). Mapping longitudinal hemispheric structural asymmetries of the human cerebral cortex from birth to 2 years of age. Cerebral Cortex, 24(5), 1289-1300. 
Lombardo, M. V., Ashwin, E., Auyeung, B., Chakrabarti, B., Taylor, K., Hackett, G., et al. (2012, Jan). Fetal testosterone influences sexually dimorphic gray matter in the human brain. The Journal of Neuroscience, 32(2), 674-680.

Lyn, H., Pierre, P., Bennett, A. J., Fears, S., Woods, R., \& Hopkins, W. D. (2011, Jun). Planum temporale grey matter asymmetries in chimpanzees (Pan troglodytes), vervet (Chlorocebus aethiops sabaeus), rhesus (Macaca mulatta) and bonnet (Macaca radiata) monkeys. Neuropsychologia, 49(7), 2004-2012.

Maingault, S., Tzourio-Mazoyer, N., Mazoyer, B., \& Crivello, F. (2016, Mar). Regional correlations between cortical thickness and surface area asymmetries: A surface-based morphometry study of 250 adults. Neuropsychologia.

Marie, D., Roth, M., Lacoste, R., Nazarian, B., Bertello, A., Anton, J.L., et al. (2018). Left brain asymmetry of the planum temporale in a nonhominid primate: Redefining the origin of brain specialization for language. Cerebral Cortex, 28(5), 1808-1815.

Meyer, M., Kircher, M., Gansauge, M. T., Li, H., Racimo, F., Mallick, S., et al. (2012, Oct). A high-coverage genome sequence from an archaic Denisovan individual. Science, 338(6104), 222-226.

Miller, J. A., Ding, S. L., Sunkin, S. M., Smith, K. A., Ng, L., Szafer, A., et al. (2014, Apr). Transcriptional landscape of the prenatal human brain. Nature, 508(7495), 199-206.

Miller, K. L., Alfaro-Almagro, F., Bangerter, N. K., Thomas, D. L., Yacoub, E., Xu, J., et al. (2016, Sep). Multimodal population brain imaging in the UK Biobank prospective epidemiological study. Nature Neuroscience, 19(11), 1523-1536.

Morch-Johnsen, L., Nesvag, R., Jorgensen, K. N., Lange, E. H., Hartberg, C. B., Haukvik, U. K., et al. (2017, Jan). Auditory cortex characteristics in schizophrenia: Associations with auditory hallucinations. Schizophrenia Bulletin, 43(1), 75-83.

Ng, B., White, C. C., Klein, H. U., Sieberts, S. K., McCabe, C., Patrick, E., et al. (2017, Oct). An XQTL map integrates the genetic architecture of the human brain's transcriptome and epigenome. Nature Neuroscience, 20(10), 1418-1426.

Norris, D. P. (2012, Dec). Cilia, calcium and the basis of left-right asymmetry. BMC Biology, 10, 102.

Ocklenburg, S., Friedrich, P., Fraenz, C., Schluter, C., Beste, C., Gunturkun, O., et al. (2018, July). Neurite architecture of the planum temporale predicts neurophysiological processing of auditory speech. Science Advances, 4, eaar6830.

Ocklenburg, S., Schmitz, J., Moinfar, Z., Moser, D., Klose, R., Lor, S., et al. (2017, Feb). Epigenetic regulation of lateralized fetal spinal gene expression underlies hemispheric asymmetries. Elife, 6.

Papadatou-Pastou, M., \& Martin, M. (2017). Cerebral laterality for language is related to adult salivary testosterone levels but not digit ratio (2D:4D) in men: A functional transcranial Doppler ultrasound study. Brain and Language, 166, 52-62.

Postema, M. C., Carrion-Castillo, A., Fisher, S. E., Vingerhoets, G., \& Francks, C. (2019). No clear monogenic links between lefthandedness and situs inversus. bioRxiv. https://doi.org/ 10.1101/422964. Retrieved from https://www.biorxiv.org/ content/early/2018/09/20/422964.

Pruim, R. J., Welch, R. P., Sanna, S., Teslovich, T. M., Chines, P. S., Gliedt, T. P., et al. (2010, Sep). LocusZoom: Regional visualization of genome-wide association scan results. Bioinformatics, 26(18), 2336-2337.

Ramasamy, A., Trabzuni, D., Guelfi, S., Varghese, V., Smith, C., Walker, R., et al. (2014, Oct). Genetic variability in the regulation of gene expression in ten regions of the human brain. Nature Neuroscience, 17(10), 1418-1428.

Ratnanather, J. T., Poynton, C. B., Pisano, D. V., Crocker, B., Postell, E., Cebron, S., et al. (2013, Nov). Morphometry of superior temporal gyrus and planum temporale in schizophrenia and psychotic bipolar disorder. Schizophrenia Research, 150(2-3), 476-483.
Reiter, J. F., \& Leroux, M. R. (2017, Sep). Genes and molecular pathways underpinning ciliopathies. Nature Reviews Molecular Cell Biology, 18(9), 533-547.

Ripke, S., Neale, B. M., Corvin, A., Walters, J. T., Farh, K. H., Holmans, P. A., et al. (2014, Jul). Biological insights from 108 schizophrenia-associated genetic loci. Nature, 511(7510), 421-427.

Rojas, D. C., Bawn, S. D., Benkers, T. L., Reite, M. L., \& Rogers, S. J. (2002, Aug). Smaller left hemisphere planum temporale in adults with autistic disorder. Neuroscience Letters, 328(3), 237-240.

Rojas, D. C., Camou, S. L., Reite, M. L., \& Rogers, S. J. (2005, Aug). Planum temporale volume in children and adolescents with autism. Journal of Autism Developmental Disorders, 35(4), 479-486.

Sabbagh, M. F., Heng, J. S., Luo, C., Castanon, R. G., Nery, J. R., Rattner, A., et al. (2018, Sep). Transcriptional and epigenomic landscapes of CNS and non-CNS vascular endothelial cells. Elife, 7.

Savage, J. E., Jansen, P. R., Stringer, S., Watanabe, K., Bryois, J., de Leeuw, C. A., et al. (2018, Jul). Genome-wide association metaanalysis in 269,867 individuals identifies new genetic and functional links to intelligence. Nature Genetics, 50(7), 912-919.

Shapleske, J., Rossell, S. L., Woodruff, P. W., \& David, A. S. (1999, Jan). The planum temporale: A systematic, quantitative review of its structural, functional and clinical significance. Brain Research Brain Research Reviews, 29(1), 26-49.

Smith, S. M., Jenkinson, M., Woolrich, M. W., Beckmann, C. F., Behrens, T. E., Johansen-Berg, H., et al. (2004). Advances in functional and structural MR image analysis and implementation as FSL. Neuroimage, 23(Suppl. 1), S208-S219.

Sommer, I., Ramsey, N., Kahn, R., Aleman, A., \& Bouma, A. (2001, Apr). Handedness, language lateralisation and anatomical asymmetry in schizophrenia: meta-analysis. The British Journal of Psychiatry, 178, 344-351.

Speed, D., \& Balding, D. (2019, Feb). SumHer better estimates the SNP heritability of complex traits from summary statistics. Nature Genetics, 51(2), 277-284.

Sudlow, C., Gallacher, J., Allen, N., Beral, V., Burton, P., Danesh, J., et al. (2015, Mar). UK biobank: An open access resource for identifying the causes of a wide range of complex diseases of middle and old age. PLoS Medicine, 12(3), e1001779.

Sun, T., Patoine, C., Abu-Khalil, A., Visvader, J., Sum, E., Cherry, T. J., et al. (2005, Jun). Early asymmetry of gene transcription in embryonic human left and right cerebral cortex. Science, 308(5729), 1794-1798.

Toga, A. W., \& Thompson, P. M. (2003, Jan). Mapping brain asymmetry. Nature Reviews Neuroscience, 4(1), 37-48.

Turner, S. (2017). Qqman: Q-Q and Manhattan plots for GWAS data. Retrieved from https://CRAN.R-project.org/package=qqman.

Tzourio-Mazoyer, N., Crivello, F., \& Mazoyer, B. (2018, Apr). Is the planum temporale surface area a marker of hemispheric or regional language lateralization? Brain Structure and Function, 223(3), 1217-1228.

Tzourio-Mazoyer, N., Maingault, S., Panzieri, J., Pepe, A., Crivello, F., \& Mazoyer, B. (2019, May). Intracortical myelination of Heschl's gyrus and the planum temporale varies with Heschl's duplication pattern and rhyming performance: An investigation of 440 healthy volunteers. Cerebral Cortex, 29(5), 2072-2083. https://doi.org/10.1093/ cercor/bhy088.

Tzourio-Mazoyer, N., \& Mazoyer, B. (2017, Aug). Variations of planum temporale asymmetries with heschl's gyri duplications and association with cognitive abilities: MRI investigation of 428 healthy volunteers. Brain Structure and Function, 222(6), 2711-2726.

Tzourio-Mazoyer, N., Simon, G., Crivello, F., Jobard, G., Zago, L., Perchey, G., et al. (2010, Jun). Effect of familial sinistrality on 
planum temporale surface and brain tissue asymmetries. Cerebral Cortex, 20(6), 1476-1485.

Vanderauwera, J., Altarelli, I., Vandermosten, M., De Vos, A., Wouters, J., \& Ghesquiere, P. (2018, Jan). Atypical structural asymmetry of the planum temporale is related to family history of dyslexia. Cerebral Cortex, 28(1), 63-72.

Verhoef, E., Demontis, D., Burgess, S., Shapland, C. Y., Dale, P. S., Okbay, A., et al. (2019, Jan). Disentangling polygenic associations between attention-deficit/hyperactivity disorder, educational attainment, literacy and language. Translational Psychiatry, 9(1), 35.

Vingerhoets, G. (2019, Oct). Phenotypes in hemispheric functional segregation? Perspectives and challenges. Physics of Life Reviews, 30, 1-18.

Visscher, P. M., Hemani, G., Vinkhuyzen, A. A., Chen, G. B., Lee, S. H., Wray, N. R., et al. (2014, Apr). Statistical power to detect genetic (co)variance of complex traits using SNP data in unrelated samples. PLoS Genetics, 10(4), e1004269.

Wada, J. A., Clarke, R., \& Hamm, A. (1975, Apr). Cerebral hemispheric asymmetry in humans. Cortical speech zones in 100 adults and 100 infant brains. Archives in Neurology, 32(4), 239-246.

Watanabe, K., Taskesen, E., van Bochoven, A., \& Posthuma, D. (2017, Nov). Functional mapping and annotation of genetic associations with FUMA. Nature Communications, 8(1), 1826.

Watkins, K. E., Paus, T., Lerch, J. P., Zijdenbos, A., Collins, D. L., Neelin, P., et al. (2001, Sep). Structural asymmetries in the human brain: A voxel-based statistical analysis of 142 MRI scans. Cerebral Cortex, 11(9), 868-877.

Westra, H. J., Peters, M. J., Esko, T., Yaghootkar, H., Schurmann, C., Kettunen, J., et al. (2013, Oct). Systematic identification of trans eQTLs as putative drivers of known disease associations. Nature Genetics, 45(10), 1238-1243.
Willems, R. M., Van der Haegen, L., Fisher, S. E., \& Francks, C. (2014, Mar). On the other hand: Including left-handers in cognitive neuroscience and neurogenetics. Nature Reviews Neuroscience, 15(3), 193-201.

Xia, M., Wang, J., \& He, Y. (2013). BrainNet viewer: A network visualization tool for human brain connectomics. PLoS One, 8(7), e68910.

Yang, J., Benyamin, B., McEvoy, B. P., Gordon, S., Henders, A. K., Nyholt, D. R., et al. (2010, Jul). Common SNPs explain a large proportion of the heritability for human height. Nature Genetics, 42(7), 565-569.

Yeni-Komshian, G. H., \& Benson, D. A. (1976, Apr). Anatomical study of cerebral asymmetry in the temporal lobe of humans, chimpanzees, and rhesus monkeys. Science, 192(4237), 387-389.

Zhang, Y., Brady, M., \& Smith, S. (2001, Jan). Segmentation of brain MR images through a hidden Markov random field model and the expectation-maximization algorithm. IEEE Transactions in Medicine Imaging, 20(1), 45-57.

Zhang, Y., Chen, K., Sloan, S. A., Bennett, M. L., Scholze, A. R., O'Keeffe, S., et al. (2014, Sep). An RNA-sequencing transcriptome and splicing database of glia, neurons, and vascular cells of the cerebral cortex. The Journal of Neuroscience, 34(36), 11929-11947.

Zhang, Y., Sloan, S. A., Clarke, L. E., Caneda, C., Plaza, C. A., Blumenthal, P. D., et al. (2016, Jan). Purification and characterization of progenitor and mature human astrocytes reveals transcriptional and functional differences with mouse. Neuron, 89(1), 37-53.

Zhernakova, D. V., Deelen, P., Vermaat, M., van Iterson, M., van Galen, M., Arindrarto, W., et al. (2017, Jan). Identification of context-dependent expression quantitative trait loci in whole blood. Nature Genetics, 49(1), 139-145. 